\title{
A Study of Community-Acquired Pneumonias in Elderly Individuals in Bijapur, India
}

\author{
Bilal Bin Abdullah, ${ }^{1}$ Mohammed Zoheb, ${ }^{1}$ Syed Mustafa Ashraf, ${ }^{2}$ \\ Sharafath Ali, ${ }^{2}$ and Nida Nausheen ${ }^{3}$ \\ ${ }^{1}$ Department of Medicine, Al Ameen Medical College \& Hospital, Karnataka, Bijapur 586108, India \\ ${ }^{2}$ Department of Internal Medicine, Al Ameen Medical College \& Hospital, Karnataka, Bijapur 586108, India \\ ${ }^{3}$ Department of Pathology, Al Ameen Medical College \& Hospital, Karnataka, Bijapur 586108, India
}

Correspondence should be addressed to Bilal Bin Abdullah, drbilal28@yahoo.com

Received 10 January 2012; Accepted 6 February 2012

Academic Editor: M. L. Metersky

Copyright (C) 2012 Bilal Bin Abdullah et al. This is an open access article distributed under the Creative Commons Attribution License, which permits unrestricted use, distribution, and reproduction in any medium, provided the original work is properly cited.

\begin{abstract}
Community-acquired pneumonia (CAP) in elderly has different clinical presentation and higher mortality than CAP in other age group. Clinical presentation may vary from mere presence of fever to altered sensorium. The incomplete clinical picture of CAP in the elderly may be associated with a delay in establishing the diagnosis and, consequently, in starting adequate antibiotic therapy. Delay in diagnosis and treatment may contribute to the higher observed death rate in the elderly population with CAP. Hence the following study was undertaken to study the clinical, radiological, and bacteriological profile of community-acquired pneumonia in elderly. A total of 50 patients were studied. Age group varied from 66 years to 88 years. Presentation varied from typical symptoms to altered sensorium. Smoking and COPD were most common predisposing conditions. Most common organisms responsible were Streptococcus pneumonia, Klebsiella pneumonia, Pseudomonas, H. influenza, and Staphylococcus aureus. Etiological agents could not be identified in many cases because of difficulty in collecting sputum in elderly patients, lower yield of culture, and various atypical and difficult to isolate causative organisms. Hence there is need for an empirical therapy covering both typical and atypical organisms. Better understanding of these aspects may help a long way in managing elderly patients with pneumonia.
\end{abstract}

\section{Introduction}

Pneumonia "the captain of men of death," "The friend of the aged, allowing them a merciful relief from those cold gradations of decay, that make the last state of all so distressing" as described by William osler, is one of the most common infectious disease encountered in the clinical practice [1].

Pneumonia is the sixth leading cause of death in the United States [2]. About 600,000 persons with pneumonia are hospitalized each year, and there are 64 million days of restricted activity due to this illness [3]. The reported incidence rates of radiologically confirmed community acquired pneumonia in different populations varied between 1.3 and 11.6 cases per 1000 inhabitant-year with highest rates in elderly adults that is $13-15$ cases per 1000 inhabitant-year. In developed countries almost one half of total hospitalization for pneumonia occur in patients over 65 years, and pneumonia is a leading cause of death in this age group [4].

Managing pneumonia in an elderly patient requires an appreciation of many aspects of geriatric medicine, including the demographics of our aging population [5]. The effect of pneumonia on the general health of an elderly person, and knowledge of how pneumonia in this population is different than inyounger populations. As stated by Sir William Osler, "In old age, pneumonia may be latent, coming on without chill, the cough and expectoration are slight, the physical signs ill defined and changeable, and the constitutional symptoms out of all proportion."

Most patients who require hospitalization for the treatment of community-acquired pneumonia (CAP) are elderly. The elderly have impaired function of many organs by virtue of the aging process and as a result of comorbidity [6]. There 
are structural and functional alterations in old age which impair the host's defense against pulmonary infection $[7,8]$.

Elderly patients hospitalized because of CAP constitute a special population and since they commonly have underlying illnesses, prior neurologic disturbances, nutritional and immunologic deficits. CAP in this population has peculiar clinical characteristics. For instance, not all the signs and symptoms of pneumonia are present in all cases. The clinical presentation may consist only of an alteration of the patient's general condition, confusion, or decompensation of underlying disease. The incomplete clinical picture of CAP in the elderly may be associated with a delay in establishing the diagnosis and, consequently, in starting adequate antibiotic therapy. Delay in diagnosis and treatment may contribute to the higher observed death rate in the elderly population with CAP $[9,10]$. Hence the following study was undertaken to study the clinical, radiological, and bacteriological profile of community-acquired pneumonia in elderly.

\section{Material and Methods}

The present study was conducted at Al-Ameen Medical College hospital and District hospital, Bijapur (affiliated to AlAmeen Medical College) between December 2008 and June 2010. This study is a prospective study carried out on 50 cases of community-acquired pneumonia of patients aged $>65$ years. Prior to the study, the protocol was approved by the institutional ethical committee, and all patients gave their informed consent to participate.

2.1. Inclusion Criteria. They are following: (a) age $>65$ years, (b) clinical symptoms like fever, cough with or without expectoration, pleuritic chest pain, dyspnea, and altered sensorium and (c) clinical signs like tachypnea, reduced chest movements, dull percussion note, bronchial breath sounds, increased vocal fremitus, and vocal resonance and crepitations. (d) Radiological evidence of pneumonia without any clinical evidence of pneumonia will also be included.

2.2. Exclusion Criteria. (a) Hospital acquired pneumonia that is patient hospitalized in the previous 14 days. (b) HIVpositive status. (c) Tuberculosis. (d) Lung malignancies.

Patient demographic features were recorded according to a standard questionnaire. A detailed clinical history was taken. History for comorbid illness and habits like smoking and alcoholism were taken. Comorbid illnesses were defined as the presence of coexisting cardiac failure, ischemic heart disease, chronic lung disease (copd), chronic liver disease (cirrhosis of liver), chronic kidney disease (diabetic nephropathy), malignancies (multiple myeloma), neurological diseases (old h/o strokes) and diabetes mellitus. A detailed clinical examination was carried out including general physical examination, vital signs, and respiratory system examination, mainly for signs of consolidation and other systemic examination for the comorbid illness. Routine investigations like hemoglobin, total leucocytes count, differential count, ESR, random blood sugar, blood urea, serum creatinine, liver function tests, and serum electrolytes
TABLe 1: Age and sex distribution.

\begin{tabular}{lcc}
\hline Age in years & No. of patients & Percentage \\
\hline $65-74$ & 32 & $64 \%$ \\
$75-84$ & 14 & $28 \%$ \\
$\geq 85$ & 4 & $8 \%$ \\
\hline Sex & No. of patients & Percentage \\
\hline Male & 35 & $70 \%$ \\
Female & 15 & $30 \%$ \\
\hline
\end{tabular}

were sent. Radiological evaluation was done. Sputum was collected for gram stain and culture and sensitivity, before starting empirical antibiotic therapy. Antibiotic was subsequently changed if necessary based on clinical response and culture and sensitivity reports.

2.3. Statistical Analysis. The data was analyzed by Mean \pm SD (standard deviation), percentage, and Chi-square test $(P$ value $<0.05$ was considered significant).

\section{Results}

The present study was conducted at Al-Ameen Medical College hospital and District hospital, Bijapur (affiliated to AlAmeen Medical College) between December 2008 and June 2010. This study is a prospective study carried out on 50 cases of hospitalized community-acquired pneumonia patients aged $>65$ years. A detailed history was taken, clinical parameters on admission were noted, routine investigations, chest Xray, and sputum examination were carried out on all patients. The results and observations of the study are as follows.

3.1. Age Distribution. In this study the age group of patients varied from 66 to 88 years. Mean age was $72.22 \pm 6.14$ years. Majority of patients were in the age group 65-74 years. The detailed age distribution is shown in Table 1.

3.2. Sex Distribution. Out of 50 patients, 35 (70\%) were males, and $15(30 \%)$ were females. The detail of sex distribution is shown in Table 1.

3.3. Predisposing Conditions. Among the predisposing conditions, COPD was the most common, noted in $24(48 \%)$ patient. Other predisposing conditions noted were diabetes mellitus in $14(28 \%)$ patients, congestive cardiac failure in 8 $(16 \%)$, neurologic diseases in $4(8 \%)$ patients, renal diseases in $3(6 \%)$ patients, chronic liver disease in $2(4 \%)$ patients, and malignancy in $1(2 \%)$ patients. Among habits, smoking was most common noted in 32 (74\%) patients followed by alcoholism in $8(16 \%)$ patients. This is depicted in Table 2.

3.4. Symptomatology. In this study patients presented with both typical and atypical symptoms. Among the typical respiratory symptoms, cough was present in 37 (74\%) patients, expectoration in $32(64 \%)$ patients, 23 had mucopurulent sputum, and 9 patients had mucoid sputum, fever in 28 
Table 2: Predisposing conditions.

\begin{tabular}{lcc}
\hline Predisposing conditions & No. of patients & Percentage \\
\hline Smoking & 32 & $74 \%$ \\
Alcoholism & 8 & $6 \%$ \\
COPD & 24 & $48 \%$ \\
Diabetes mellitus & 14 & $24 \%$ \\
Congestive cardiac failure & 8 & $16 \%$ \\
Neurologic diseases & 4 & $8 \%$ \\
Renal diseases & 3 & $6 \%$ \\
Chronic liver diseases & 2 & $4 \%$ \\
Malignancy & 1 & $2 \%$ \\
\hline
\end{tabular}

TABle 3: Table showing symptomatology.

\begin{tabular}{lcc}
\hline Presenting symptoms & No. of patients & Percentage \\
\hline Cough & 37 & $74 \%$ \\
Expectoration & 32 & $64 \%$ \\
Fever & 28 & $56 \%$ \\
Dyspnea & 11 & $22 \%$ \\
Pleuritic chest pain & 10 & $20 \%$ \\
Altered sensorium & 8 & $16 \%$ \\
Gastrointestinal symptoms & 4 & $8 \%$ \\
\hline
\end{tabular}

TABLE 4: General physical examination.

\begin{tabular}{lcc}
\hline Signs & No. of patients & Percentage \\
\hline Pallor & 13 & $26 \%$ \\
Icterus & 6 & $12 \%$ \\
Cyanosis & 4 & $8 \%$ \\
Clubbing & 2 & $4 \%$ \\
Pedal oedema & 4 & $8 \%$ \\
\hline
\end{tabular}

(56\%) patients, dyspnea in $11(22 \%)$, and pleuritic chest pain in $10(20 \%)$ patients. Among the atypical symptoms altered sensorium was present in $8(16 \%)$ patients and gastrointestinal symptoms of anorexia, nausea, vomiting, or diarrhea in $4(8 \%)$ patients. The details of symptomatology is shown in Table 3.

3.5. Findings on General Physical Examination. General physical examination of the patient showed pallor in 13 (26\%) patients, icterus in $6(12 \%)$ patients, cyanosis in 4 (8\%) patients, clubbing in $2(4 \%)$ and pedal oedema in 4 (8\%) patients. This is shown in Table 4.

3.6. Vital Signs. In this study, raised temperature $>38^{\circ} \mathrm{C}$ was noted in $34(68 \%)$ patients, in 18 patients it was $38.9 \mathrm{c}-$ $39.9 \mathrm{c}$, and 14 patients had $39.9 \mathrm{c}-40 \mathrm{c}$, tachypnea defined by respiratory rate $>24 / \mathrm{min}$ was noted in $42(84 \%)$ patients ( 25 had respiratory rate of $24-26 / \mathrm{min}$ and 17 patients had respiratory rate of $27-30 / \mathrm{min}$ ), tachycardia defined as pulse rate $>100 / \mathrm{min}$ was noted in $35(70 \%)$ patients (28 patients had pulse rate in range of $100-120 / \mathrm{min}$ and 7 patients
TABLE 5: Vital signs.

\begin{tabular}{lcc}
\hline Vital signs & Present study & Percentage \\
\hline Temperature $>38 \mathrm{c}$ & 34 & $68 \%$ \\
Tachypnea $>24 / \mathrm{min}$ & 42 & $84 \%$ \\
Tachycardia $>100 / \mathrm{min}$ & 35 & $70 \%$ \\
Hypotension $<90 \mathrm{mmHg}$ & 8 & $16 \%$ \\
Temperature $<35 \mathrm{c}$ & 1 & $2 \%$ \\
\hline
\end{tabular}

TABLE 6: Respiratory system examination findings.

\begin{tabular}{lcc}
\hline Clinical signs & No. of patients & Percentage \\
\hline Reduced chest movements & 10 & $20 \%$ \\
Impaired note & 14 & $28 \%$ \\
Bronchial breathing & 12 & $24 \%$ \\
Increased VF and VR & 10 & $20 \%$ \\
Crepitations & 47 & $94 \%$ \\
Pleural rub & 12 & $24 \%$ \\
\hline
\end{tabular}

had pulse rate of $120-130 / \mathrm{min}$ ), hypotension defined as systolic blood pressure $<90 \mathrm{mmHg}$ was noted in $8(16 \%)$ patients (6 patients had SBP of $60-80 \mathrm{~mm} \mathrm{Hg}$ and SBP was not recordable in 2 patients) and hypothermia defined as temperature $<35^{\circ} \mathrm{C}$ was noted in $1(2 \%)$ patients (34.6c). This is shown in Table 5.

3.7. Findings on Respiratory System Examination. In this study, crepitation was the most common respiratory system examination findings, which was noted in 47 (94\%) patients. Other findings were reduced chest movements, impaired note on percussion, bronchial breathing, increased vocal fremitus and vocal resonance, and pleural rub. The details of these are shown in Table 6.

3.8. Laboratory Characteristics. Leucocytosis defined as total leucocyte count $>11,000 /$ cum was the most common, noted in $42(84 \%)$ patients. Leucopenia defined as total leucocyte count $<4,000 /$ cumm was noted in $4(8 \%)$ patients. Anemia defined as $\mathrm{Hb}<11 \mathrm{gm} / \mathrm{dL}$ was noted in $16(32 \%)$ patients. ESR $>20 \mathrm{~mm}$ at 1 hour was noted in $38(76 \%)$ patients. Blood urea $>40 \mathrm{mg} / \mathrm{dL}$ was noted in $20(40 \%)$ patients. Serum creatinine $>1.4 \mathrm{mg} / \mathrm{dL}$ was noted in $8(16 \%)$ patients. Raised serum bilurubin greater $>1.2 \mathrm{mg} / \mathrm{dL}$ was noted in $6(12 \%)$, and raised liver enzymes were noted in $8(16 \%)$ patients. Hypoalbuminemia defined as serum albumin $<3.5 \mathrm{mg} / \mathrm{dL}$ was noted in $14(28 \%)$, patients. Hyponatremia defined by serum sodium $<130 \mathrm{meq} / \mathrm{L}$ was noted in $14(28 \%)$ and hypokalemia was noted in $2(4 \%)$ patients. Details of these are shown in Table 7.

3.8.1. Results of Sputum Gram Staining. Adequate sputum sample showing bacteriological positivity could be obtained in only $26(54 \%)$ patients. Of these, $16(32 \%)$ were grampositive cocci, $6(12 \%)$ were gram-negative bacilli, and 4 $(8 \%)$ were mixed. Details are shown in Table 8. 
TABLE 7: Laboratory parameters.

\begin{tabular}{|c|c|c|}
\hline Laboratory parameters & No. of patients & Percentage \\
\hline Anemia $<11$ g/dL $(13-15 \mathrm{~g} / \mathrm{dL})$ & 16 & $32 \%$ \\
\hline Leucocytosis $>11,000 /$ cumm $(5000-10,0000 /$ cumm $)$ & 42 & $84 \%$ \\
\hline Leucopenia $<4,000 /$ cumm & 4 & $8 \%$ \\
\hline \multicolumn{3}{|l|}{ Raised ESR $>20 \mathrm{~mm}$ at $1 \mathrm{hr}$} \\
\hline Age $\quad 20 \quad 55 \quad 90$ & \multirow{3}{*}{38} & \multirow{3}{*}{$76 \%$} \\
\hline $\begin{array}{lll}12 & 14 & 19\end{array}$ & & \\
\hline Women $\quad 18 \quad 21 \quad 23$ & & \\
\hline High RBS > 200 mg/dL (90-130 mg/dL) & 8 & $16 \%$ \\
\hline Raised urea >40 mg/dL (20-40 mg/dL) & 20 & $40 \%$ \\
\hline Serum creatinine $>1.4 \mathrm{mg} / \mathrm{dL}(0.6-1.5 \mathrm{mg} / \mathrm{dL})$ & 8 & $16 \%$ \\
\hline Raised bilurubin >1.2 mg/dL (0.2-1 mg/dL) & 6 & $12 \%$ \\
\hline \multicolumn{3}{|l|}{ Raised liver enzymes } \\
\hline SGPT-9-40 IU/L & 8 & $16 \%$ \\
\hline \multicolumn{3}{|l|}{ SGOT-10-35 IU/L } \\
\hline Hypoalbuminemia (3.5-5.3 g/L) & 14 & $28 \%$ \\
\hline Hyponatremia (135-145 mEq/L) & 8 & $16 \%$ \\
\hline Hypokalemia (3.5-5.0 mEq/L) & 2 & $4 \%$ \\
\hline
\end{tabular}

TABLE 8: Sputum gram staining.

\begin{tabular}{lcc}
\hline Gram staining & No. of patients & Percentage \\
\hline Gram-positive cocci & 16 & $32 \%$ \\
Gram-negative bacilli & 6 & $12 \%$ \\
Mixed & 4 & $8 \%$ \\
Undetermined & 24 & $48 \%$ \\
\hline
\end{tabular}

TABLE 9: Showing bacteria isolated on culture.

\begin{tabular}{lcc}
\hline Bacteria isolated & No. of patients & Percentage \\
\hline Streptococcus pneumonia & 8 & $16 \%$ \\
Klebsiella pneumonia & 3 & $6 \%$ \\
Pseudomonas & 2 & $4 \%$ \\
Hemophilus influenza & 2 & $4 \%$ \\
Staphylococcus aureus & 1 & $2 \%$ \\
Escherasia coli & 1 & $2 \%$ \\
\hline
\end{tabular}

TABLE 10: Radiological findings.

\begin{tabular}{lcc}
\hline Radiological finding & No. of patients & Percentage \\
\hline Lobar pneumonia & 39 & $78 \%$ \\
Bronchopneumonia & 9 & $18 \%$ \\
Interstitial pneumonia & 2 & $4 \%$ \\
Pleural effusion & 6 & $12 \%$ \\
Cavitation & 2 & $4 \%$ \\
\hline
\end{tabular}

3.8.2. Results of Sputum Culture. In this study positive sputum culture was obtained in only 18 patients $(32 \%)$. Streptococcus pneumonia was the most common organism isolated, obtained in 8 (16\%) patients, followed by Klebsiella pneumonia in $3(6 \%)$ patients, Pseudomonas in 2 (4\%), Hemophilus influenza in 2 (4\%), Staphylococcus aureus in 1 (2\%), and E. coli in 1 (2\%) patients. A detail of this is shown in Table 9.

\section{Sputum Gram Staining and Culture Demonstrated Correlation in 18 of 26 Patients (with Both Gram Staining and Culture Positivity and 8 Patients Had Sterile Cultures)}

4.1. Radiological Findings. In this study, lobar pneumonia was the most common radiological finding, which was noted in $39(78 \%)$ patients of which 26 patients had mid to lower zone consolidation, 8 patients had whole lung consolidation, 2 patients had upper zone consolidation and 3 had bilateral pneumonia. Out of 39 patients with lobar pneumonia the distribution was 24 to the right lung, 12 to left lung and 3 bilateral. Bronchopneumonia was noted in $9(18 \%)$ patients and interstitial pneumonia in $2(4 \%)$ patients, pleural effusion in $6(12 \%)$ patients and cavitation in $2(4 \%)$ patients. This is shown in Table 10.

4.2. Complications. The most common complication noted was septic shock in $8(16 \%)$ patients, followed by pleural effusion in $6(12 \%)$ patients. The other complications noted were congestive cardiac failure, ARDS, lung abscess, and emphysema. Details of this are shown in Table 11.

4.3. Mortality. In this study, out of 50 patients, $42(84 \%)$ patients improved and $8(16 \%)$ patients had mortality. This is shown in Table 12. 
TABLE 11: Complications.

\begin{tabular}{lcc}
\hline Complications & No. of patients & Percentage \\
\hline Septic shock & 8 & $16 \%$ \\
Pleural effusion & 6 & $12 \%$ \\
CCF & 4 & $8 \%$ \\
ARDS & 2 & $4 \%$ \\
Lung abscess & 2 & $4 \%$ \\
Emphyema & 2 & $4 \%$ \\
\hline
\end{tabular}

TABLE 12: Mortality.

\begin{tabular}{lcc}
\hline Outcome & No. of patients & Percentage \\
\hline Improved & 42 & $84 \%$ \\
Died & 8 & $16 \%$ \\
\hline
\end{tabular}

\section{Analysis of Prognostic Factors of Community Acquired Pneumonia in Elderly}

Analysis of clinical characters shows that age distribution above 65 years had no association with mortality. Among the clinical presentation altered sensorium had statistically highly significant association with increased mortality. Presence of associated diseases had statistically significant association with mortality. This is shown in Table 13.

Analysis of vital signs shows statistically significant association of temperature $>38^{\circ} \mathrm{C}$ and $<35^{\circ} \mathrm{C}$, pulse rate $>100 / \mathrm{min}$, and systolic blood pressure $<90 \mathrm{mmHg}$ with mortality. This is shown in Table 14.

Analysis of laboratory parameters shows statistically significant association of total leucocyte count $>11,000$ and $<4000$ cells/cumm, raised urea and hyponatremia with mortality, whereas raised serum creatinine, and hypoalbuminemia did not show significant association. This is shown in Table 15.

Analysis of complications shows highly significant association of ARDS and septic shock with mortality. This is shown in Table 16.

\section{Discussion}

Community-acquired pneumonia (CAP) is a frequent cause of hospital admission and mortality in elderly patients worldwide. The clinical presentation, etiology, and outcome of community-acquired pneumonia in elderly differs from that of other population. In the present study, 50 patients of community-acquired pneumonia $>65$ years of age were included. The results of study which has been presented in previously are discussed below.

6.1. Age. Pneumonia is common in the extremes of age. In this study, the age group of patients presenting with community-acquired pneumonia ranged from 66 to 88 years. Of these patients $32(64 \%)$ were aged 65-74 years, $14(28 \%)$ were aged $75-84$ years, and $4(8 \%)$ were aged $\geq 85$ years. In a study conducted by Riquelme et al. 14,069 Medicare patients age $>65$ years who required hospitalization for CAP were studied. They noted that $30.3 \%$ were aged $65-74$ years, $41.8 \%$ were aged $75-84$ years, and $27.8 \%$ were aged $>85$ years [11].

In another study by Torres et al., the mean age of elderly patients presenting with community-acquired pneumonia was $78 \pm 8$ yr (mean \pm SD) [12]. These differences may be due to differences in the rate of hospitalization and access to health care of the elderly in the different populations studied.

The increased incidence of pneumonia in elderly patients is due to the defects in mechanical clearance of airways, loss of elastic recoil of lungs, decreased strength of respiratory muscle causing decreased effectiveness of coughing, agerelated decline in mucociliary clearance, defects in humoral and cell-mediated immunity, and cumulative effects of comorbid chronic diseases and their treatments.

6.2. Sex Distribution. In this study males 35 (70\%) were affected more than females 15 (30\%). This sex distribution is similar to study conducted by Riquemele et al. where $67 \%$ were males and $33 \%$ were females, and this may be attributed to increased rates of alcoholism and smoking in males and also due to the increased association of comorbid conditions like COPD, congestive cardiac failure and others in males. This is shown in Table 17.

6.3. Predisposing Conditions. The presence of chronic comorbidity in most of the CAP patients in our study is compatible with the contention that an important risk factor for pneumonia in elderly people is the prevalence of other illnesses [13]. This is shown in Table 18.

Kobashi et al. studied 83 patients with elderly pneumonia, and thirty-eight patients (45\%) had a history of respiratory disease, with chronic obstructive pulmonary disease (COPD) being most common (16 cases). Sixty-two patients (74\%) had a history of nonrespiratory disease, most commonly cerebrovascular disease (16 patients). Fifty patients were current smoker (60\%), and 15 drank alcohol (16\%) regularly [14].

Smoking was the most important risk factor (72\%) in our study. The increased risk of pneumonia in smokers is due to alteration in respiratory flora, mechanical clearance, and cellular defenses. Bacterial colonization of lower respiratory tract is more prevalent in smokers than nonsmokers, mucociliary clearance is defective in smokers, owing to a reduction in ciliary beat frequency and changes in volume and viscoelastic properties of respiratory secretions. In a population-based case-control study, Nuorti et al. found that cigarette smoking was the strongest independent risk factor for invasive pneumococcal disease [15].

Alcoholism is also a risk factor as it interferes with various respiratory tract defenses such as alterations in normal flora, increased risk of aspiration, impaired mechanical clearance and deficient humoral and cellular immunity.

In our study COPD was most common associated comorbid condition (48\%). Increased incidence and mortality of pneumonia have been described in COPD patients. This is explained due to defective mucociliary clearance, mucous plugging, airway collapse, respiratory muscle fatigue, and the effect of medications used [16]. 
TABLE 13: Association of clinical characteristics with mortality.

\begin{tabular}{|c|c|c|c|c|c|c|}
\hline Variables & Survivors $(n=42)$ & Nonsurvivors $(n=8)$ & Total $(n=50)$ & $X^{2}$ & $P$ value & Inference* \\
\hline \multicolumn{7}{|l|}{ Age in yrs } \\
\hline $65-75$ & 28 & 4 & 32 & 3.763 & $>0.05$ & NA \\
\hline $75-85$ & 12 & 2 & 14 & & & \\
\hline$>85$ & 2 & 2 & 4 & & & \\
\hline Fever & 27 & 6 & 33 & 0.344 & 0.558 & NA \\
\hline Cough & 33 & 4 & 37 & 2.851 & 0.091 & NA \\
\hline Expectoration & 24 & 4 & 28 & 0.139 & 0.709 & NA \\
\hline Dyspnea & 9 & 2 & 11 & 0.05 & 0.823 & NA \\
\hline Pleuritic chest pain & 10 & 0 & 10 & 2.024 & 0.154 & NA \\
\hline Altered sensorium & 6 & 6 & 12 & 13.581 & 0.000 & $\mathrm{HA}$ \\
\hline Gastrointestinal symptoms & 3 & 2 & 5 & 2.381 & $>0.05$ & NA \\
\hline Associated diseases & 26 & 7 & 33 & 21.176 & 0.05 & A \\
\hline Smoking & 25 & 7 & 32 & 2.283 & 0.131 & NA \\
\hline
\end{tabular}

*NA: not associated, A: associated, HA: highly associated.

TABLE 14: Association of vital signs with mortality.

\begin{tabular}{|c|c|c|c|c|c|c|}
\hline Variables & Survivors $(n=42)$ & Nonsurvivors $(n=8)$ & Total $(n=50)$ & $X^{2}$ & $P$ value & Inference* \\
\hline \multicolumn{7}{|l|}{ Temperature } \\
\hline $35^{\circ} \mathrm{C}$ & 0 & 1 & 1 & 6.725 & 0.05 & A \\
\hline $35-38^{\circ} \mathrm{C}$ & 16 & 1 & 17 & & & \\
\hline$>38^{\circ} \mathrm{C}$ & 26 & 6 & 32 & & & \\
\hline Respiratory rate $>24 / \mathrm{min}$ & 32 & 8 & 40 & 2.381 & 0.123 & NA \\
\hline Pulse rate $>100 / \mathrm{min}$ & 28 & 8 & 36 & 3.704 & 0.054 & A \\
\hline Systolic blood pressure $<90 \mathrm{mmHg}$ & 1 & 7 & 8 & 36.226 & 0.000 & $\mathrm{HA}$ \\
\hline
\end{tabular}

${ }^{*}$ NA: not associated, A: associated, HA: highly associated.

TABLE 15: Association of laboratory data with mortality.

\begin{tabular}{|c|c|c|c|c|c|c|}
\hline Variables & Survivors $(n=42)$ & Nonsurvivors $(n=8)$ & Total $(n=50)$ & Chi-square & $P$ value & Inference* \\
\hline \multicolumn{7}{|l|}{ Total leucocyte count } \\
\hline$<4000 /$ cumm & 2 & 3 & 5 & 8.162 & 0.025 & HA \\
\hline $4000-11,000$ cumm & 5 & 1 & 6 & & & \\
\hline$>11,000$ cumm & 35 & 4 & 39 & & & \\
\hline Blood urea $>40 \mathrm{mmHg}$ & 27 & 8 & 35 & 4.082 & 0.043 & A \\
\hline Serum creatinine $>1.4 \mathrm{~g} / \mathrm{dL}$ & 22 & 7 & 29 & 3.402 & 0.065 & NA \\
\hline Hypoalbuminemia & 11 & 3 & 14 & 0.426 & 0.514 & NA \\
\hline Hyponatremia & 3 & 5 & 8 & 15.322 & 0.000 & $\mathrm{HA}$ \\
\hline
\end{tabular}

*NA: not associated, A: associated, HA: highly associated.

TABle 16: Association of complications with mortality.

\begin{tabular}{|c|c|c|c|c|c|c|}
\hline Complications & Survivors $(n=42)$ & Nonsurvivors $(n=8)$ & Total $(n=50)$ & Chi-square & $P$ value & Inference* \\
\hline ARDS & 0 & 2 & 2 & \multirow{6}{*}{43.8} & \multirow{6}{*}{0.005} & \multirow{6}{*}{ Highly significant } \\
\hline Septic shock & 2 & 6 & 8 & & & \\
\hline $\mathrm{CCF}$ & 4 & 0 & 4 & & & \\
\hline Emphyema & 2 & 0 & 2 & & & \\
\hline Lung abscess & 2 & 0 & 2 & & & \\
\hline Pleural effusion & 6 & 0 & 6 & & & \\
\hline
\end{tabular}


TABLE 17: Sex distribution in study conducted by us and Riquemele et al.

\begin{tabular}{lcc}
\hline Sex & Our study & Riquemele et al. \\
\hline Male & $70 \%$ & $67 \%$ \\
Female & $30 \%$ & $33 \%$ \\
\hline
\end{tabular}

TABLE 18: Predisposing factors seen in our study and another study conducted by Torres et al. is as follows.

\begin{tabular}{lcc}
\hline Predisposing conditions & Our study & Torres et al. \\
\hline Smoking & $32(74 \%)$ & $62(62 \%)$ \\
Alcoholism & $8(6 \%)$ & $22(22 \%)$ \\
COPD & $24(48 \%)$ & $30(30 \%)$ \\
Diabetes mellitus & $14(24 \%)$ & $1(17 \%)$ \\
Congestive cardiac failure & $8(16 \%)$ & $28(28 \%)$ \\
Neurologic diseases & $4(8 \%)$ & $5(5 \%)$ \\
Renal diseases & $3(6 \%)$ & $4(4 \%)$ \\
Chronic liver diseases & $2(4 \%)$ & $4(4 \%)$ \\
Malignancy & $1(2 \%)$ & $5(5 \%)$ \\
\hline
\end{tabular}

6.4. Presenting Complaints. Elderly patient may present with typical as well as atypical symptoms. Atypical symptoms are commonly described in elderly than in younger patients. The clinical presentation may consist of only an alteration of the patient's general condition, confusion, or decompensation of underlying disease. Clinical presentation according to our study as well as studies conducted by Torres et al. and Kobashi et al. is shown in Table 19.

In this study patients presented with atypical presentations like altered sensorium, nausea, vomiting, diarrhea, loss of appetite, and breathlessness in addition to typical symptoms of cough, expectoration, fever, and pleuritic chest pain. Cough was the most common respiratory symptom noted in $37(74 \%)$ patients, which was productive in only $29(58 \%)$ patients, due to decreased ability of elderly to bring out the sputum.

Harper and Newton found that the classical constellation of CAP in the elderly of cough, fever, and dyspnea was absent in $56 \%$ of patients [17]. Fever was the presenting feature in 32 (64\%) patients in our study. The frequency of fever in elderly patients with CAP according to various studies ranges from $33 \%$ to $60 \%$.

Altered sensorium was the presenting feature in $8(16 \%)$ of patients in our study. Venkatesan and coworkers [18] and Harper and associates found a prevalence of $47 \%$ and $15 \%$, respectively, of mental alteration in elderly patients with CAP at admission. A practical conclusion of clinical interest is that an effort has to be made to take chest radiographs, upon arrival at the hospital, of all elderly patients with delirium.

6.5. General Physical Examination Findings. In this study general physical examination of the patient showed pallor in $8(16 \%)$ patients which may be secondary to infection, associated comorbid conditions, and malnutrition. Icterus was observed in $6(12 \%)$ patients. Icterus is commonly described
TABle 19: Clinical presentation according to our study as well as studies conducted by Torres et al. and Kobashi et al.

\begin{tabular}{lccc}
\hline Presenting symptoms & Our study & Torres et al. & Kobashi et al. \\
\hline Cough & $37(74 \%)$ & $407(81 \%)$ & $52(62 \%)$ \\
Expectoration & $32(64 \%)$ & $331(66 \%)$ & $47(56 \%)$ \\
Fever & $28(56 \%)$ & $380(76 \%)$ & $47(56 \%)$ \\
Dyspnea & $11(22 \%)$ & $351(70 \%)$ & $16(19 \%)$ \\
Pleuritic chest pain & $10(20 \%)$ & $218(43 \%)$ & $12(14 \%)$ \\
Altered sensorium & $8(16 \%)$ & $130(26 \%)$ & $7(8 \%)$ \\
$\begin{array}{l}\text { Gastrointestinal } \\
\text { symptoms }\end{array}$ & $4(8 \%)$ & $36(8 \%)$ & $7(8 \%)$ \\
\hline
\end{tabular}

in streptococcal pneumonia infections and also in others as part of multiorgan dysfunction and also due to underlying chronic liver disease in some patients. Central cyanosis observed in $4(8 \%)$ patients. Central cyanosis is explained due to shunting of blood through consolidated lung. Vital signs noted in our study as well as in study conducted by Kobashi et al. is shown in Table 20.

In our study tachypnea was the most common sign 42 (84\%) patients, followed by tachycardia 35 (70\%) patients. Fever the most frequently noted symptom of infection occurs less frequently in elderly. In our study fever was noted in 34 $(68 \%)$ of patients. Poor febrile response was correlated to decrease release of IL-1 in elderly patients [19]. Hypothermia is noted in $1(2 \%)$ patient and is associated with poor prognosis. Septic shock secondary to pneumonia is commonly seen in elderly, and this may account for hypotension seen in $8(16 \%)$ of patients.

6.6. Findings of Respiratory System Examination. Among the respiratory signs noted in our study crepitations was the most frequent finding noted in most of the patients 47 (94\%). In a study by Zalcain et al., crepitations were the most common respiratory finding noted in $79 \%$ of patients [20]. Other characteristics respiratory signs like bronchial breathing, increased vocal fremitus and vocal resonance, and impaired note on percussion were present in less than one third of the patients. This shows the paucity of characteristic clinical signs in elderly patients with pneumonia and the need to diagnose pneumonia in elderly patients with atypical presentations and minimal signs and symptoms.

6.7. Laboratory Data. Among the laboratory investigations in our study, leucocytosis was the most consistent finding noted in $42(84 \%)$ patients where as leucopenia was seen in $4(8 \%)$ patient and was associated with poor prognosis. In study by Torres et al., seventy-six (76\%) patients had a leukocyte count $>10.000 / \mathrm{mm} 3$. Elevated white blood cell counts in elderly people may be caused by the increased rate of pneumococcal infection in old age.

In our study raised blood urea was noted in $20(40 \%)$ patients. Raised bilirubin in $6(12 \%)$ and raised liver enzymes in $8(16 \%)$ was noted. The hepatic impairment and the impaired renal function were more severe in the elderly age group compared to the younger groups in various studies. 
TABLE 20: Vital signs noted in our study as well as in study conducted by Kobashi et al.

\begin{tabular}{lcc}
\hline Vital signs & Present study & Kobashi et al. \\
\hline Temperature $>38 \mathrm{c}$ & $34(68 \%)$ & $47(56 \%)$ \\
Tachypnea $>24 / \mathrm{min}$ & $42(84 \%)$ & $59(70 \%)$ \\
Tachycardia $>100 / \mathrm{min}$ & $35(70 \%)$ & $58(69 \%)$ \\
Hypotension $<90 \mathrm{mmHg}$ & $8(16 \%)$ & $15(18 \%)$ \\
Temperature $<35 \mathrm{c}$ & $1(2 \%)$ & $1(1 \%)$ \\
\hline
\end{tabular}

TABLE 21: Radiological findings in our study and study by Torres et al.

\begin{tabular}{lcc}
\hline Radiological findings & Our study & Torres et al. \\
\hline Lobar pneumonia & $39(78 \%)$ & $367(82 \%)$ \\
Bronchopneumonia & $9(18 \%)$ & $62(12 \%)$ \\
Interstitial pneumonia & $2(4 \%)$ & $18(4 \%)$ \\
Pleural effusion & $6(12 \%)$ & $60(11 \%)$ \\
Cavitation & $2(4 \%)$ & $9(2 \%)$ \\
\hline
\end{tabular}

This may suggest a more severe systemic infection in this age group [21].

Hyponatremia was seen in $8(16 \%)$ of patients. Hyponatraemia due to inappropriate antidiuretic hormone $(\mathrm{ADH})$ secretion may occur in any pneumonia and is notably more common in Legionella infection.

In our study, serum albumin level was low in $14(28 \%)$ patients. Serum albumin level is commonly cited as a marker of nutritional status, and it has been correlated independently with a higher case-fatality rate among persons with CAP [22]. Although serum albumin level is an indicator of nutritional status, experimental and clinical data indicate that in inflammatory disorders the synthesis of acute-phase proteins occurs at the expense of albumin, and thus a low serum albumin level can be caused by both malnutrition and the acute inflammatory response.

In a study conducted by Kobashi et al., the main laboratory data at admission were as follows: the white blood cell count was above $10,000 /$ cumm in $58 \%$, the ESR was over $20 \mathrm{~mm}$ in one hour in $95 \%$, the, serum protein was under $6.5 \mathrm{~g} / \mathrm{dL}$ in $30 \%$, serum albumin was under $3.5 \mathrm{~g} / \mathrm{dL}$ in $35 \%$, and blood urea nitrogen (BUN) was over $20 \mathrm{mg} / \mathrm{dL}$ in $23 \%$.

6.8. Sputum Microscopy and Culture. In this study bacteriological positivity on gram staining of sputum was seen in 26 (52\%) of patients. Cultures were positive in only $18(32 \%)$ patients. Streptococcus pneumonia was the most common etiological agent isolated, $8(16 \%)$ patients, followed by gram-negative bacilli like Klebsiella pneumonia 3 (6\%), Pseudomonas aeruginosa 2 (4\%), E. coli 1 (2\%), Hemophilus influenza $2(4 \%)$ and Staphylococcus aureus 1 (2\%) patient. This is almost similar to study conducted by Torres et al., where Sputum analysis was performed in 403 (80\%) cases and of these $186(46 \%)$ were of good quality and 71 (38\%) showed positive results, bacteriological diagnosis was achieved in 11 of 30 cases (37\%), with 13 microorganisms (five S. pneumoniae, two C. pneumoniae, one L. pneumophila, one $P$. aeruginosa, one Klebsiella pneumoniae, one Escherichia coli, one Staphylococcus aureus, one Streptococcus viridians.

Woodhead et al. reviewed 11 studies that reported on the etiology of pneumonia in the elderly and compared them to 3 studies of pneumonia in younger populations. The proportion of cases due to $H$. influenzae, $S$. aureus, and gram-negative bacilli was higher among the elderly, and the proportion due to Legionella and other atypical pathogens was higher among the younger patients [23].

Colonization of the oropharyngeal mucosa with aerobic gram negative Bacilli like Escherichia coli, Klebsiella species, Proteus species, Enterobacter species, and Pseudomonas aeruginosa increases with increasing age, predisposing to increased incidence of gram-negative bacilli in aged [24].

In various studies in Western counties undertaken to investigate the causes of community-acquired pneumonia in the elderly, the distribution of causative pathogens appeared to differ from that in younger adults, S. pneumoniae, was the most common causing about $50 \%$ of infections, respiratory viruses, $H$. influenzae, gram-negative bacilli, C. pneumoniae and L. pneumophila were others commonly noted [25].

The cause of CAP is often difficult to establish. The most effective methods are often invasive and cannot always be justified, and serological diagnosis is too late to be of any therapeutic use. Despite the progress made in the diagnosis of pneumonia, it takes a few days to identify the causative microorganism in the blood or sputum samples, and the etiology of half of all patients with CAP remains uncertain [26]. Physicians need reliable data on the relative prevalence of different etiological agents in the patients' area of residence, in addition to the clinical, laboratory, and radiological findings in order to initiate antibiotic treatment empirically. The relative frequency of etiological agents varies among different geographical areas [27].

6.9. Radiological Presentation. The radiological data in our study showed a predominance of lobar pneumonia in 39 (78\%) patients followed by bronchopneumonia in $9(18 \%)$ patients. Radiological data of our study compared with that due to Torres et al. is shown in Table 21 .

Riquelme et al. found the chest-radiographic infiltrate pattern on admission as alveolar in $82 \%$ cases interstitial in $6 \%$ and mixed in $13 \%$ and unilateral in $71 \%$.

6.10. Complications. Various complications noted in our study are pleural effusion $6(12 \%)$, septic shock $8(6 \%)$, ARDS 3 (6\%), lung abscess 2 (4\%), emphyema $2(4 \%)$, and decompensation of heart failure $4(8 \%)$ patients. 
Zalacain et al. noted pleural effusion in $60(12 \%)$, septic shock in $41(8 \%)$, lung abscess in $2(4 \%)$, and emphysema in $14(3 \%)$ as common complications in their study.

6.11. Mortality. In this study, $8(16 \%)$ patients had mortality. Mortality rates for elderly patients hospitalized with CAP in previous reports range from 6 to $40 \%$ [28]. Along with asso-ciated comorbidities and malnutrition, increased age itself is a independent risk factor for increased mortality for CAP in elderly. Kaplan et al. reported a mortality of $11 \%$ in elderly patients with community-acquired pneumonia [29].

6.12. Prognostic Factors. In our study, the following prognostic factors shown to have statistically significant association with mortality; altered sensorium, presence of associated diseases, temperature $>38^{\circ} \mathrm{C}$ or $<35^{\circ} \mathrm{C}$, pulse rate $>100 / \mathrm{min}$, systolic blood pressure $<90 \mathrm{mmHg}$, leucocytosis $>11,000$ cells/cumm, leucopenia $<4000$ cells/cumm, raised urea $>40 \mathrm{mg} / \mathrm{dL}$, hyponatremia, ARDS and septic shock.

Conte et al. developed a prognostic rule for elderly patients admitted with CAP. They found five predictors of bad prognosis as follows: presence of comorbidity, abnormal vital signs axillary temperature $>38^{\circ} \mathrm{C}$, cardiac frequency $>110$ beats/min and systolic arterial pressure $<90 \mathrm{mmHg}$, age $>85 \mathrm{yrs}$, alteration in mental state, and plasma creatinine $>1.5 \mathrm{mg} / \mathrm{dL}[30]$.

\section{Conclusion}

Community-acquired pneumonia in elderly patients is a common and serious problem encountered in clinical practice. Elderly patients with community-acquired pneumonia have different clinical presentation and higher mortality. Elderly patients may present with atypical symptoms like altered sensorium and gastrointestinal symptoms, other than the typical respiratory symptoms of pneumonia. They may not have all the classical signs of consolidation and may present with only few signs like tachypnea, tachycardia, and crepitations. The atypical presentations may lead to delay in diagnosis and initiation of treatment and may be responsible for higher observed mortality in elderly patients with pneumonia.

Etiological agents cannot be identified in many cases because of difficulty in collecting sputum in elderly patients, lower yield of culture, and various atypical and difficult to isolate causative organisms. Hence the need for an empirical therapy covering both typical and atypical organisms.

The following prognostic factors shown to have statistically significant association with mortality; altered sensorium, presence of associated diseases, temperature $>38^{\circ} \mathrm{C}$ or $<35^{\circ} \mathrm{C}$, pulse rate $>100 / \mathrm{min}$, systolic blood pressure $<90 \mathrm{mmHg}$, leucocytosis $>11,000$ cells/cumm, leucopenia $<4000$ cells/cumm, raised urea $>40 \mathrm{mg} / \mathrm{dL}$, hyponatremia, ARDS and septic shock. Prospective studies on larger number of patients are required to substantiate these findings.

\section{References}

[1] S. L. Berk, "Bacterial pneumonia in the elderly: the observations of Sir William Osler in retrospect," Journal of the American Geriatrics Society, vol. 32, no. 9, pp. 683-685, 1984.

[2] R. E. Dixon, "Economic costs of respiratory tract infections in the United States," American Journal of Medicine, vol. 78, no. 6, pp. 45-51, 1985.

[3] National Centre for Health Statistics, "National hospital discharge survey: annual summary 1990," Vital Health Statistics, vol. 13, pp. 1-225, 1992.

[4] O. Ochoa-Gondar, A. Vila-Córcoles, C. De Diego et al., "The burden of community-acquired pneumonia in the elderly: the Spanish EVAN-65 Study," BMC Public Health, vol. 8, article 222, 2008

[5] A. M. Medina-Walpole and P. R. Katz, "Nursing homeacquired pneumonia," Journal of the American Geriatrics Society, vol. 47, no. 8, pp. 1005-1015, 1999.

[6] B. A. Cunha, D. Gingrich, and G. S. Rosenbaum, "Pneumonia syndromes: a clinical approach in the elderly," Geriatrics, vol. 45, no. 10, pp. 49-55, 1990.

[7] A. M. Fein, S. H. Feinsilver, and M. S. Niederman, "Atypical manifestations of pneumonia in the elderly," Clinics in Chest Medicine, vol. 12, no. 2, pp. 319-336, 1991.

[8] J. T. Granton and R. F. Grossman, "Community-acquired pneumonia in the elderly patient: clinical features, epidemiology, and treatment," Clinics in Chest Medicine, vol. 14, no. 3, pp. 537-553, 1993.

[9] P. Venkatesan, J. Gladman, J. T. Macfarlane et al., "A hospital study of community acquired pneumonia in the elderly," Thorax, vol. 45, no. 4, pp. 254-258, 1990.

[10] T. P. Meehan, M. J. Fine, H. M. Krumholz et al., "Quality of care, process, and outcomes in elderly patients with pneumonia," Journal of the American Medical Association, vol. 278, no. 23, pp. 2080-2084, 1997.

[11] T. P. Meehan, M. J. Fine, H. M. Krumholz et al., "Quality of care, process, and outcomes in elderly patients with pneumonia," Journal of the American Medical Association, vol. 278, no. 23, pp. 2080-2084, 1997.

[12] R. Riquelme, A. Torres, M. El-Ebiary et al., "Communityacquired pneumonia in the elderly. Clinical and nutritional aspects," American Journal of Respiratory and Critical Care Medicine, vol. 156, no. 6, pp. 1908-1914, 1997.

[13] A. M. Fein and M. S. Niederman, "Severe pneumonia in the elderly," Clinics in Geriatric Medicine, vol. 10, no. 1, pp. 121143, 1994.

[14] Y. Kobashi, N. Okimoto, T. Matsushima, and R. Soejima, "Clinical analysis of community-acquired pneumonia in the elderly," Internal Medicine, vol. 40, no. 8, pp. 703-707, 2001.

[15] J. P. Nuorti, J. C. Butler, M. M. Farley et al., "Cigarette smoking and invasive pneumococcal disease," New England Journal of Medicine, vol. 342, no. 10, pp. 681-689, 2000.

[16] A. Torres, J. Dorca, R. Zalacaín et al., "Community-acquired pneumonia in chronic obstructive pulmonary disease: a Spanish multicenter study," American Journal of Respiratory and Critical Care Medicine, vol. 154, no. 5, pp. 1456-1461, 1996.

[17] C. Harper and P. Newton, "Clinical aspects of pneumonia in the elderly veteran," Journal of the American Geriatrics Society, vol. 37, no. 9, pp. 867-872, 1989.

[18] P. Venkatesan, J. Gladman, J. T. Macfarlane et al., "A hospital study of community acquired pneumonia in the elderly," Thorax, vol. 45, no. 4, pp. 254-258, 1990. 
[19] C. A. Kauffman, P. G. Jones, and M. J. Kluger, "Fever and malnutrition, endogenous pyrogen, interleukin-1 in malnourished patients," American Journal of Clinical Nutrition, vol. 44, no. 4, pp. 449-452, 1986.

[20] R. Zalacain, A. Torres, R. Celis et al., "Community-acquired pneumonia in the elderly: Spanish multicentre study," European Respiratory Journal, vol. 21, no. 2, pp. 294-302, 2003.

[21] W.-S. Lim and J. T. Macfarlane, "Defining prognostic factors in the elderly with community acquired pneumonia: a case controlled study of patients aged $\geq 75$ yrs," European Respiratory Journal, vol. 17, no. 2, pp. 200-205, 2001.

[22] J. Hedlund, L. O. Hansson, and A. Ortqvist, "Short- and longterm prognosis for middle-aged and elderly patients hospitalized with community-acquired pneumonia: impact of nutritional and inflammatory factors," Scandinavian Journal of Infectious Diseases, vol. 27, no. 1, pp. 32-37, 1995.

[23] M. Woodhead, "Pneumonia in the elderly," Journal of Antimicrobial Chemotherapy, vol. 34, pp. 85-92, 1994.

[24] W. M. Valenti, R. G. Trudell, and D. W. Bentley, "Factors predisposing to oropharyngeal colonization with gram-negative bacilli in the aged," New England Journal of Medicine, vol. 298, no. 20, pp. $1108-1111,1978$.

[25] M. S. Niederman and A. M. Fein, "Community-acquired Pneumonia in the Elderly," in Respiratory Infections in the Elderly, pp. 44-72, Raven Press, New York, NY, USA, 1991.

[26] T. Ishida, T. Hashimoto, M. Arita, I. Ito, and M. Osawa, "Etiology of community-acquired pneumonia in hospitalized patients: a 3-year prospective study in Japan," Chest, vol. 114, no. 6, pp. 1588-1593, 1998.

[27] D. Lieberman, F. Schlaeffer, I. Boldur et al., "Multiple pathogens in adult patients admitted with community-acquired pneumonia: a one year prospective study of 346 consecutive patients," Thorax, vol. 51, no. 2, pp. 179-184, 1996.

[28] J. T. Granton and R. F. Grossman, "Community-acquired pneumonia in the elderly patient: clinical features, epidemiology, and treatment," Clinics in Chest Medicine, vol. 14, no. 3, pp. 537-553, 1993.

[29] V. Kaplan, D. C. Angus, M. F. Griffin, G. Clermont, R. Scott Watson, and W. T. Linde-Zwirble, "Hospitalized communityacquired pneumonia in the elderly: age- and sex-related patterns of care and outcome in the United States," American Journal of Respiratory and Critical Care Medicine, vol. 165, no. 6, pp. 766-772, 2002.

[30] H. A. Conte, Y. T. Chen, W. Mehal, D. Phil, J. D. Scinto, and V. J. Quagliarello, "A prognostic rule for elderly patients admitted with community-acquired pneumonia," American Journal of Medicine, vol. 106, no. 1, pp. 20-28, 1999. 


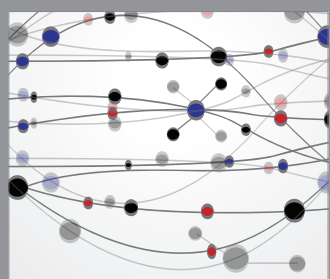

The Scientific World Journal
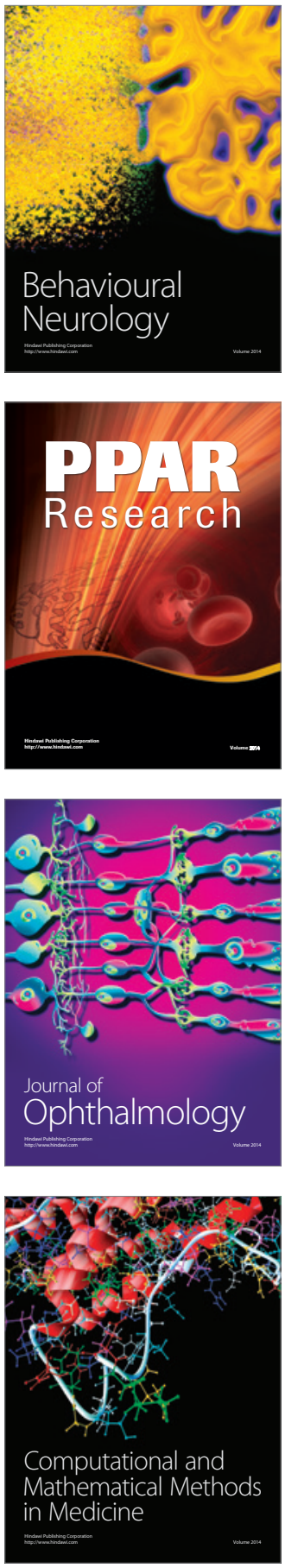

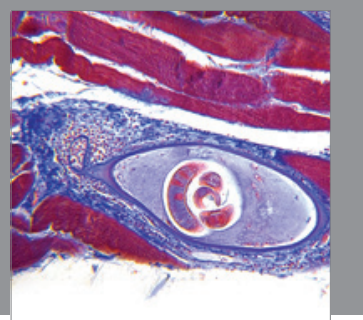

Gastroenterology

Research and Practice
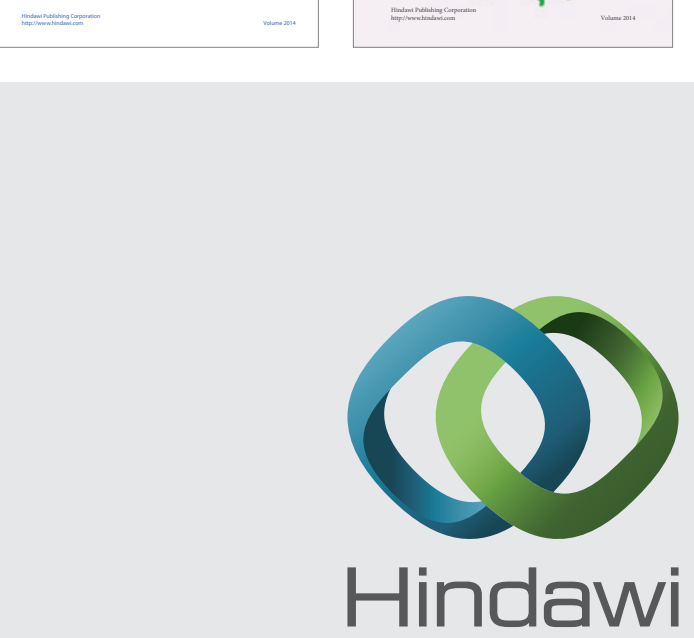

Submit your manuscripts at

http://www.hindawi.com
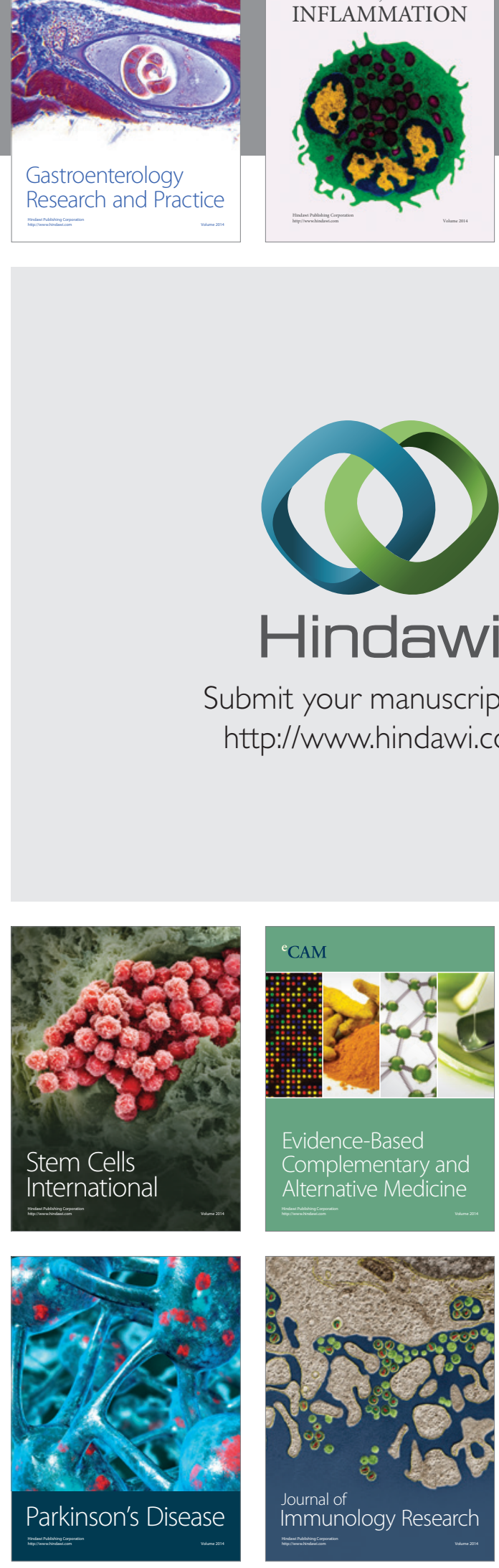

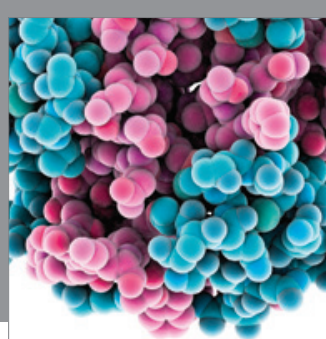

Diabetes Research
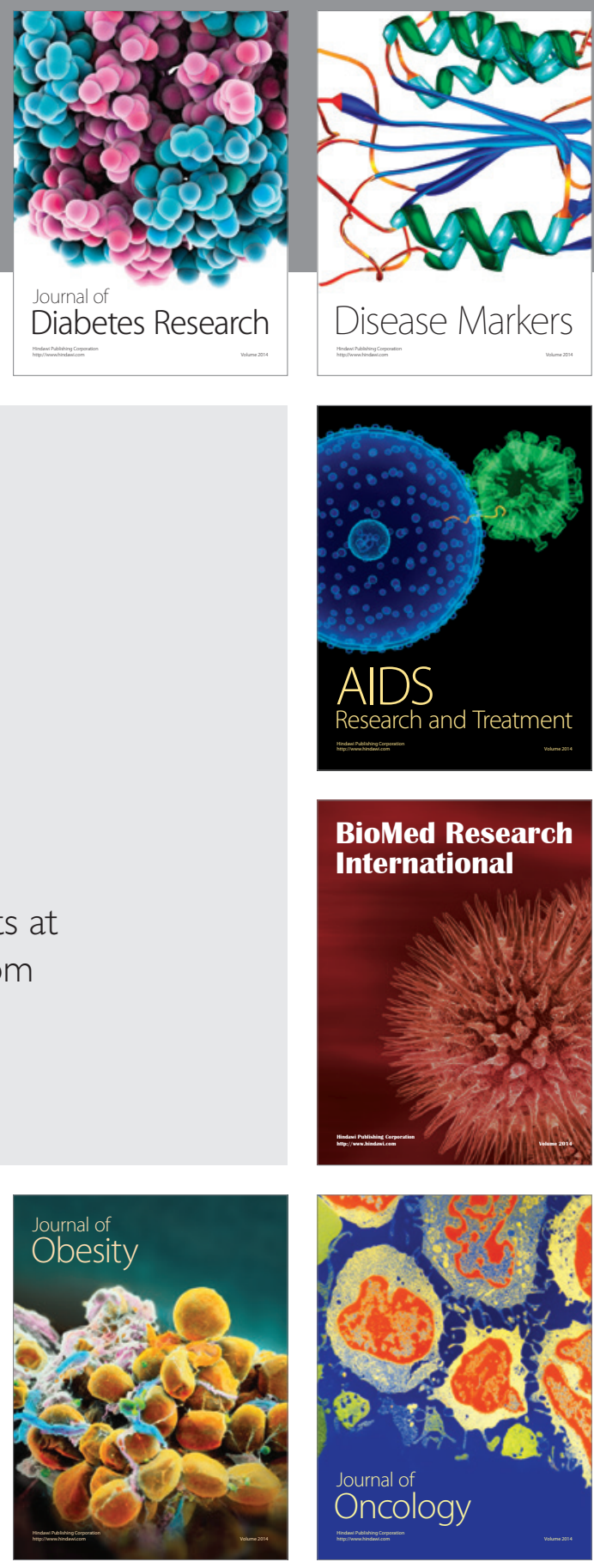

Disease Markers

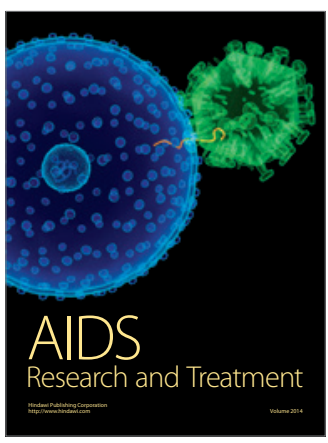

BioMed Research

International
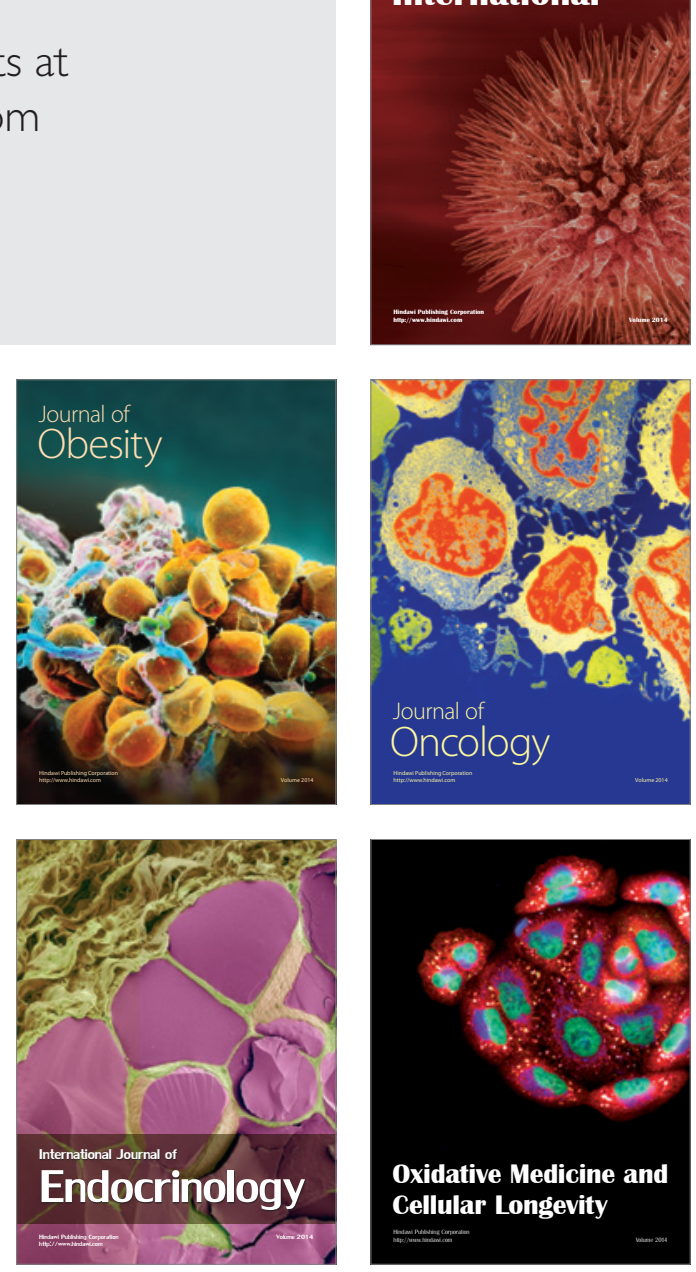